Behavioral/Cognitive

\title{
Perceptual Learning at a Conceptual Level
}

\author{
Rui Wang, ${ }^{1,2 *}$ Jie Wang, ${ }^{1 *}$ Jun-Yun Zhang, ${ }^{2 *}$ Xin-Yu Xie, ${ }^{2 *}$ Yu-Xiang Yang, ${ }^{2}$ Shu-Han Luo, ${ }^{1}$ Cong Yu, ${ }^{2}$ and Wu Li ${ }^{1}$ \\ ${ }^{1}$ State Key Laboratory of Cognitive Neuroscience and Learning and IDG/McGovern Institute for Brain Research, Beijing Normal University, Beijing 100875, \\ China, and ${ }^{2}$ Department of Psychology, IDG/McGovern Institute for Brain Research, and Peking-Tsinghua Center for Life Sciences, Peking University \\ 100871 Beijing, China
}

Humans can learn to abstract and conceptualize the shared visual features defining an object category in object learning. Therefore, learning is generalizable to transformations of familiar objects and even to new objects that differ in other physical properties. In contrast, visual perceptual learning (VPL), improvement in discriminating fine differences of a basic visual feature through training, is commonly regarded as specific and low-level learning because the improvement often disappears when the trained stimulus is simply relocated or rotated in the visual field. Such location and orientation specificity is taken as evidence for neural plasticity in primary visual cortex (V1) or improved readout of V1 signals. However, new training methods have shown complete VPL transfer across stimulus locations and orientations, suggesting the involvement of high-level cognitive processes. Here we report that VPL bears similar properties of object learning. Specifically, we found that orientation discrimination learning is completely transferrable between luminance gratings initially encoded in V1 and bilaterally symmetric dot patterns encoded in higher visual cortex. Similarly, motion direction discrimination learning is transferable between first- and second-order motion signals. These results suggest that VPL can take place at a conceptual level and generalize to stimuli with different physical properties. Our findings thus reconcile perceptual and object learning into a unified framework.

Key words: perceptual learning; motion direction; orientation; transfer

\section{Significance Statement}

Training in object recognition can produce a learning effect that is applicable to new viewing conditions or even to new objects with different physical properties. However, perceptual learning has long been regarded as a low-level form of learning because of its specificity to the trained stimulus conditions. Here we demonstrate with new training tactics that visual perceptual learning is completely transferrable between distinct physical stimuli. This finding indicates that perceptual learning also operates at a conceptual level in a stimulus-invariant manner.

\section{Introduction}

One of the remarkable functions of the brain is its capability to learn from past experience to improve cognitive and perceptual skills. Some forms of visual learning, such as recognition and categorization of complex objects, lead to abstraction of the rules defining the critical features of the object category (Bruner et al.,

Received July 19, 2015; revised Dec. 10, 2015; accepted Jan. 9, 2016.

Author contributions: J.-Y.Z.,C.Y., and W.L. designed research; R.W., J.W., X.-Y.X.,Y.-X.Y., and S.-H.L. performed research; R.W., J.W., J.-Y.Z., X.-Y.X., C.Y., and W.L. analyzed data; C.Y. and W.L. wrote the paper.

This work was supported by National Key Basic Research Program of China (Grant 2014CB846101) and the National Natural Science Foundation of China (Grants 31230030, 31470975, and 91432102). We thank Gerald Westheimer and Rufin Vogels for valuable comments.

The authors declare no competing financial interests.

*R.W., J.W., J.-Y.Z., and X.-Y.X. contributed equally to this work.

Correspondence should be addressed to either of the following: Cong Yu, Department of Psychology, Peking

University, Beijing 100871, China, E-mail: yucong@pku.edu.cn; or Wu Li, State Key Laboratory of Cognitive Neuroscience and Learning, Beijing Normal University, Beijing 100875, China, E-mail: liwu@bnu.edu.cn.

DOI:10.1523/JNEUROSCI.2732-15.2016

Copyright $\odot 2016$ the authors $\quad 0270-6474 / 16 / 362238-09 \$ 15.00 / 0$
1956; Rouder and Ratcliff, 2006). As a result, the learning effect can be generalized to a broad range of previously unseen objects that belong to the same learned category but are different in physical properties. Such cognitive ability is also important for invariant recognition of the same object under diverse viewing conditions. Conversely, visual perceptual learning (VPL)-improvement in discriminating subtle differences in basic visual features such as the orientation of a line or the moving direction of a dot- has long been regarded as a unique learning form because it is highly specific to the training conditions (Fahle, 2002). For example, the discrimination threshold (i.e., the justnoticeable difference) for a stimulus's orientation or moving direction is much reduced with training, but this learning effect usually disappears when the same stimulus is placed at a new visual field location or when its orientation/moving direction is rotated by 90 degrees (Crist et al., 1997). Such learning specificity coincides with the coding strategy in the primary visual cortex (V1), where different neurons represent different visual field lo- 
cations, stimulus orientations, and moving directions (Hubel and Wiesel, 1959, 1962). Therefore, VPL is often interpreted as training-induced changes specific to the subset of V1 neurons encoding the trained stimulus (Karni and Sagi, 1991; Schoups et al., 1995; Teich and Qian, 2003), or as improved readout of V1 sensory signals specifically activated by the trained stimulus (Mollon and Danilova, 1996; Dosher and Lu, 1999; Law and Gold, 2009).

However, our previous studies have shown that the location and orientation/direction specificity of VPL can be eliminated with new double-training or training-plus-exposure (TPE) procedures (Xiao et al., 2008; J.Y. Zhang et al., 2010; Zhang and Yang, 2014; Xiong et al., 2016) and that the learning effect can even transfer to the opposite visual field represented by the untrained brain hemisphere (Wang et al., 2012). For example, Vernier learning can transfer to a new location or orientation completely if the observers receive additional exposure of the new location or orientation via performing an irrelevant task (Wang et al., 2012, 2014; J.Y. Zhang et al., 2014). These results cannot be easily explained by specific changes in early visual cortical areas that are topographically organized to represent the visual field locations and to encode simple stimulus attributes such as line orientations and dot moving directions. Instead, a more central mechanism has to be introduced to account for the transfer of VPL.

The transferability of VPL is usually examined by comparing the same physical stimulus under the trained versus untrained conditions. It remains unknown whether the learning effect could generalize between different physical stimuli that define the same visual feature (e.g., a specific orientation or motion direction). This issue is particularly important because such learning transfer would suggest that VPL can take place at a conceptual level, similar to category or object learning. In this study, we investigated this possibility with the classical VPL tasks of orientation and motion direction discrimination.

\section{Materials and Methods}

Observers and apparatus. Seventy-four naive observers (33 male and 41 female) with normal or corrected-to-normal vision were recruited from undergraduate and graduate students. All except four in a control experiment (see Fig. $5 G$ ) were inexperienced. They were required to sign an informed consent form before the study. All experimental procedures conformed to the Declaration of Helsinki and were approved by the ethics committees of Beijing Normal University and Peking University.

The stimuli were generated with a MATLAB-based Psychotoolbox-3 (Pelli, 1997) and presented on a 21 inch Sony G520 color monitor (1024 pixel $\times 768$ pixel, $0.38 \mathrm{~mm} \times 0.38 \mathrm{~mm}$ per pixel, $120 \mathrm{~Hz}$ frame rate). The mean luminance was $50 \mathrm{~cd} / \mathrm{m}^{2}$. The luminance of the monitor was linearized by an 8-bit look-up table. Viewing was binocular through a circular opening (diameter $=17^{\circ}$ ) of a piece of black cardboard that covered the rest of the monitor screen. A chin-and-head rest helped stabilize the head of the observer. Experiments were run in a dimly lit room. An Eyelink II eye tracker (SR Research) was used to monitor eye movements in a control experiment (see Fig. 5F).

Visual stimuli. The stimuli used for orientation discrimination training in the fovea included Gabor and noise grating patches and bilaterally symmetric dot patterns (see Fig. $1 A$ ). The Gabor gratings (Gaussian-enveloped sinusoidal gratings) were set at $1.5 \mathrm{cycles} /{ }^{\circ}$ and $50 \%$ contrast, with the phase randomized for every presentation. The $\mathrm{SD}$ of the Gaussian envelop was $0.67^{\circ}$. The noise gratings (Schoups et al., 1995) consisted of pixelated stripes within a circular field ( $3^{\circ}$ diameter). The widths and spacing of the stripes randomly varied between $0.09^{\circ}$ and $0.37^{\circ}$ and were re-randomized for each presentation.

The symmetric dot patterns consisted of 18 pairs of bilaterally symmetric white dots $\left(0.1^{\circ}\right.$ diameter $)$, which were confined to an area divided into $18 \times 18$ invisible square compartments $\left(0.16^{\circ} \times 0.16^{\circ}\right.$ each $)$. The placement of the 18 dots on one side of the symmetry axis (within 18 rows by 9 columns of available compartments) was subject to the following constraints: (1) no dot was placed in the column of compartments nearest to the symmetry axis; (2) for the other 8 columns, 2 of them were randomly chosen to hold 3 dots in each column and each of the remaining 6 columns contained 2 dots; (3) only one dot was allowed in each of the 18 rows by randomly assigning row numbers to the 18 dots on one side of the symmetric pattern; and (4) the location of each dot was randomly jittered by $0-0.04^{\circ}$ from the compartment center. After positioning the 18 dots on one side of the symmetry axis, the whole symmetric pattern was generated by placing another 18 mirror-imaged dots on the other side. For each stimulus presentation, the dot pattern was regenerated, preventing the observers from using local cues in the orientation discrimination task.

The stimuli used for motion direction discrimination in the fovea included luminance-modulated (i.e., first-order) stimuli and contrastmodulated (i.e., second-order) stimuli that were replicated from a previous study (Petrov and Hayes, 2010). In brief, a first-order stimulus was an additive combination of a radially isotropic moving modulator and a dynamic noise carrier, and a second-order stimulus was a multiplicative combination of the same moving modulator and noise carrier. The modulator had a diameter of $6.5^{\circ}$, contrast of 1 , and moving speed of $4 \%$. The edge of the modulator was blurred with a semitransparent linear ramp from $5.5^{\circ}$ to $6.5^{\circ}$ in diameter.

Experimental procedure. In the orientation, motion direction, luminance, and contrast discrimination tasks, the thresholds were measured in a temporal 2-alternative forced-choice task using a 3-down 1-up staircase procedure that converged at a $79.4 \%$ correct response rate.

In each trial, the reference and test stimuli were randomly presented in two intervals (orientation or luminance task: $106 \mathrm{~ms}$ each; direction or contrast task: $800 \mathrm{~ms}$ ) separated by an interstimulus interval (orientation or luminance: $500 \mathrm{~ms}$; direction or contrast: $200 \mathrm{~ms}$ ). For the orientation and motion direction discrimination tasks, the reference stimulus was set at $35^{\circ}$ or $125^{\circ}$ and the test stimulus was deviated from the reference by an amount automatically controlled by the staircase procedure. The observer's task was to judge in which interval the stimulus was tilted more clockwise. For the mean luminance discrimination task with the dot pattern stimuli, the luminance of individual dots was randomized within $\pm 20 \%$ of a specific mean luminance level. This level was chosen in such a way that the mean luminance of the entire reference pattern was fixed at $80 \%$ of the screen maximal luminance. The mean luminance of the entire test pattern, which was lower, was controlled by a staircase. The observer's task was to report in which interval the dot pattern had a higher mean luminance. For the contrast discrimination task with the second-order motion stimuli, the contrast of the modulator in the reference stimulus was set at 1.00, and the contrast of the test stimulus was decreased by an amount controlled by a staircase. The observer's task was to report in which interval the stimulus had a higher contrast.

Auditory feedback was given on incorrect responses. Each staircase consisted of 10 reversals ( $\sim 50-60$ trials). The geometric mean of the last six reversals was taken as the discrimination threshold. The pretraining and posttraining thresholds were calculated based on six staircases. The mean thresholds in a training session (day) were calculated based on 16 or 10 staircases, as indicated in the text.

In a motion adaptation experiment (see Fig. $5 G$ ), the second-order motion stimulus at one of the training directions was first presented for 1 $\mathrm{min}$ as an adaptor at $5^{\circ}$ retinal eccentricity left to the fixation point. It was then presented for $4 \mathrm{~s}$ at the beginning of each subsequent trial. After a $200 \mathrm{~ms}$ delay, a pair of identical first-order or second-order motion stimuli were presented for $1.5 \mathrm{~s}$, one at the same adapted location and the other at a mirrored location in the opposite visual hemifield. The observers compared the speeds of the two stimuli by reporting which one moved faster. A method of constant stimuli was used to measure the perceived speed at the adapted location. The speed of the stimulus at the adapted location was the same as the adaptor speed. The speed of the other stimulus varied in seven levels from trial to trial. Each level consisted of 48 trials. The point of subjective equality (PSE) at 50\% response rate was estimated by fitting the psychometric function with a 
A Symmetric dots
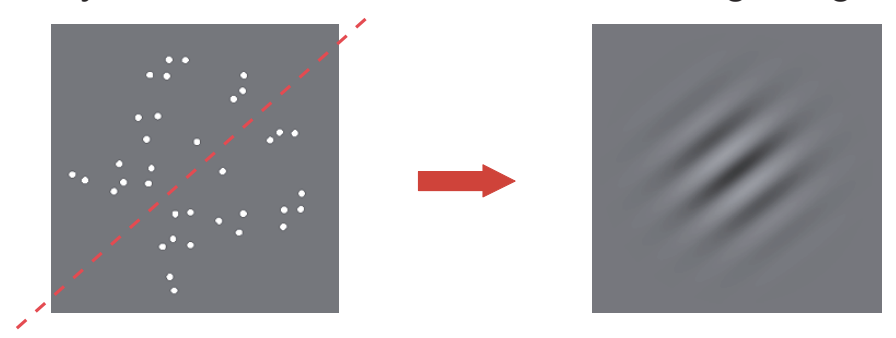

B

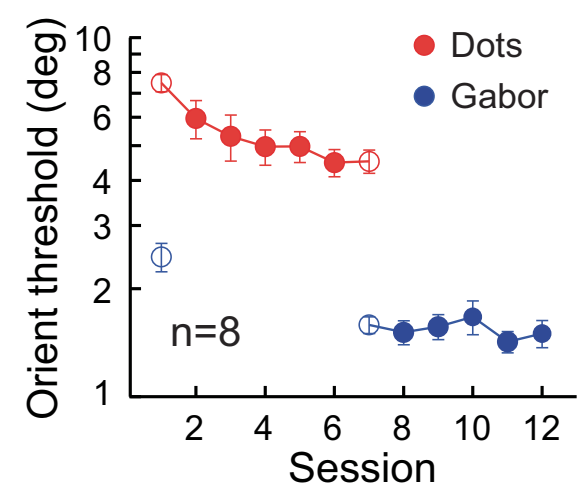

Gabor grating

\section{Noise grating}

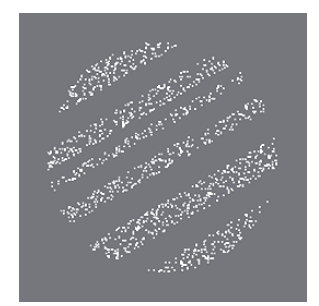

Figure 1. Transfer of orientation discrimination learning from symmetric dot patterns to gratings. $\boldsymbol{A}$, Sample stimuli. The symmetry axis is indicated by the red dashed line (not shown in the actual stimuli). The arrow indicates the direction of learning transfer. B, Session-by-session mean discrimination thresholds for dot pattern orientation ("Dots") and grating orientation ("Gabor" or "Noise"). Grating orientation discrimination was tested with Gabor gratings (left) and noise gratings (right). C, Summary of dot pattern orientation learning and its transfer to grating orientation ("Training1" and "Transfer"; left two bars) and the impact of further grating orientation training ("Training2"; right bar). Data are averaged over the two panels in $\boldsymbol{B}$. The percentage improvement was calculated as (pretraining threshold - posttraining threshold)/pretraining threshold. Error bars indicate \pm SEM.

Weibull function. Control data with no adaptors were also collected as the baselines.

\section{Results}

In the orientation discrimination task, human observers were trained to discriminate a small difference in orientation between two otherwise identical stimuli that were displayed briefly and successively. The stimuli were either luminance gratings or bilaterally symmetric dot patterns (Fig. 1A). Processing of these two types of oriented stimuli is known to engage different visual cortical areas. The grating orientations are initially encoded in V1 (Hubel and Wiesel, 1959, 1962), the earliest stage of visual information processing in the cortex, whereas the symmetric dot patterns only selectively activate higher-order cortical areas (Sasaki et al., 2005; Tyler et al., 2005). Two types of luminance gratings were used. One type was conventional Gabor gratings (sinusoidal gratings with a Gaussian envelope), which match the receptive field profiles of V1 simple cells well (Watson et al., 1983). Because the periodicity of the sinusoidal component of Gabor gratings might contain symmetry information around their orientation axis, we also tested with "noise gratings" formed by pixelated stripes of different widths and spacing (Schoups et al., 1995). These noise gratings should activate symmetry detectors minimally. The experimental results were similar for both types of grating stimuli and were thus pooled for data analyses.

We first investigated whether orientation discrimination learning could transfer from symmetric dot patterns to gratings (Fig. 1A). Fourteen observers practiced orientation discrimination when the symmetry axis of the dot patterns was set at $35^{\circ}$ or $125^{\circ}$ (counterbalanced across observers). Five sessions of practice on different days (16 blocks of trials in each training session) reduced the discrimination threshold by $42.8 \pm 3.1 \%(p<0.001$, two-tailed paired $t$ test in this and later analyses unless stated otherwise; Cohen's $d=3.62$; Fig. $1 B, C)$. After training with the dot patterns, orientation discrimination of Gabor and noise gratings around the same axis also showed significant improvement $(35.1 \pm 3.4 \%, p<0.001$, Cohen's $d=2.78$; Fig. $1 B, C)$. The improvement rates for the trained (dot patterns) and untrained (gratings) stimuli were not statistically different from each other $(p=0.12$, Cohen's $d=0.44)$, indicating significant transfer of the learning effect.

To determine whether the learning transfer was complete, the observers further practiced the orientation discrimination task with the grating stimuli for another five sessions. This subsequent training only led to a very small and insignificant improvement of orientation discrimination $(5.4 \pm 3.5 \%, p=0.15$, Cohen's $d=0.41$; Fig. $1 B, C)$, indicating that the earlier learning transfer was nearly complete. It is noteworthy that the pretraining and posttraining thresholds for the global dot patterns were two to three times larger than those for the gratings. The complete learning transfer thus suggests that the learning takes place at a central processing stage, where orientation signals defined by physically distinct stimuli are analyzed in a similar way even if these stimuli are initially encoded by separate neural mechanisms with different levels of precision.

In contrast, in the reverse direction (Fig. $2 A$ ), the transfer of orientation discrimination learning from gratings to dot patterns first appeared to be only partial. Another 14 naive observers practiced grating orientation discrimination for five sessions on different days at either $35^{\circ}$ or $125^{\circ}$, which improved the performance by $40.1 \pm$ 
A Gabor grating

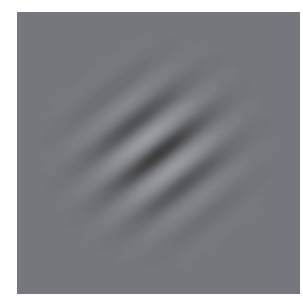

Noise grating

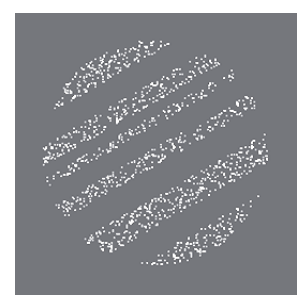

Symmetric dots

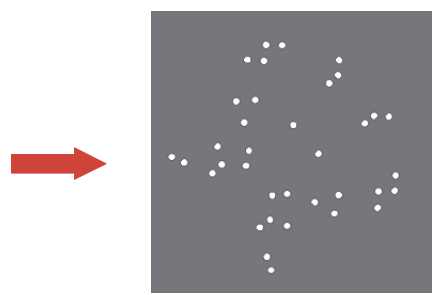

B

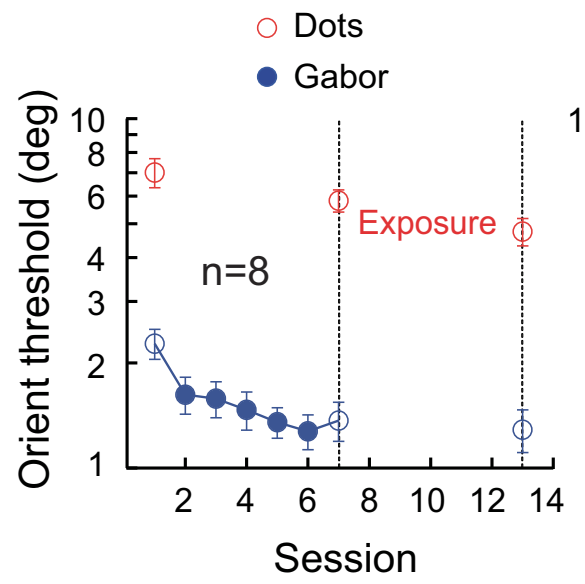

$\circ$ Dots

- Noise

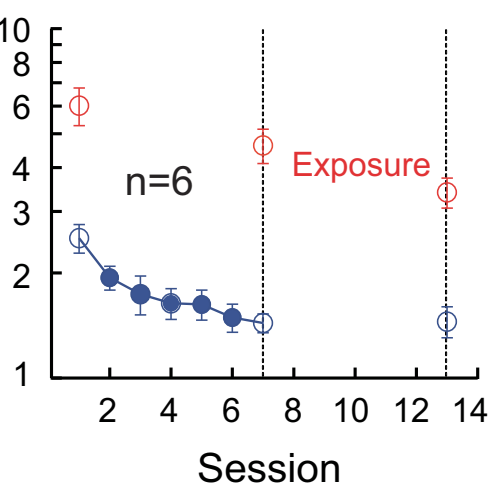

C

Figure 2. Transfer of orientation discrimination learning from gratings to symmetric dot patterns. $\boldsymbol{A}$, Sample stimuli. The arrow indicates the direction of transfer. $\boldsymbol{B}$, Session-by-session thresholds in a sequential TPE procedure in which orientation discrimination training with the gratings ("Gabor" and "Noise") was followed by exposure with symmetric dot patterns ("Dots"). Grating orientation discrimination was tested with Gabor gratings (left) and noise gratings (right). C, Summary of grating orientation learning and its direct transfer to dot patterns ("Training1" and "Transfer"; left two bars); additional transfer after the dot pattern exposure and the overall transfer ("Exposure"; middle two bars); and the impact of further direct training on global pattern orientation discrimination ("Training2"; right bar). Error bars indicate \pm SEM.

$3.3 \%(p<0.001$, Cohen's $d=3.24$; Fig. $2 B, C)$. Training with the gratings also led to improved discriminability for the dot patterns by $16.4 \pm 5.2 \%(p=0.007$, Cohen's $d=0.85$; Fig. $2 B, C)$, but at a rate that was less than half of the improvement when the dot patterns were trained directly (Fig. 1).

Previously, we applied double-training and TPE techniques to enable substantial, and often complete, learning transfer to untrained stimulus locations and orientations/directions (Xiao et al., 2008; J.Y. Zhang et al., 2010; Zhang and Yang, 2014; Xiong et al., 2016). Therefore, we suspected that similar techniques could also be effective for the current learning tasks. To test this possibility, we had the same observers subsequently exposed to the same-oriented symmetric dot patterns by performing an easy task (mean accuracy 98\%) that was irrelevant to orientation discrimination. In this task, the observers judged which of the two consecutively displayed patterns contained more dots, with one pattern having the same number of dots as in the previous experiments and the other having only $2 / 3$ of the dots. After this exposure phase ( 5 sessions, 16 blocks of 50 trials per session), dot pattern orientation discrimination was further improved by $21.8 \pm 2.4 \%(p<0.001$, Cohen's $d=2.38$; Fig. $2 B, C)$. The overall improvement amounted to $35.0 \pm 4.1 \%(p<0.001$, Cohen's $d=2.27$; Fig. $2 B, C$ ), not significantly different from the improvement with direct training shown in Figure 1, $B$ and $C$ ( $p=0.18$, two-tailed independent $t$ test; Cohen's $d=0.38)$. To determine whether the learning transfer was complete after the exposure phase, we called 10 of the 14 observers back to further practice dot pattern orientation discrimination for five sessions. This direct training only slightly improved the performance by
$7.6 \pm 2.8 \%(p=0.024$, Cohen's $d=0.86$; Fig. $2 C)$, indicating that prior grating orientation learning had mostly transferred to dot patterns after applying the TPE procedure.

In addition to the above sequential TPE procedure, we also tested 5 more observers in a simultaneous TPE procedure in which training in grating orientation discrimination and the exposure of symmetric dot patterns were conducted within the same sessions in alternating block of trials ( 10 blocks each) for 5 sessions (Fig. 3). This procedure required only one pretest of dot pattern orientation threshold (before grating orientation training), unlike the previous sequential TPE procedure, in which two pretests of dot pattern orientation threshold (one before grating training, the other before dot pattern exposure) might have confounded the transfer effect. Grating orientation learning $(40.2 \pm 3.8 \%, p<0.001$, Cohen's $d=2.87)$ again mostly transferred to the dot patterns $(36.9 \pm 4.2 \%, p<0.001$, Cohen's $d=2.37$ ). Five additional sessions of training in dot pattern orientation discrimination only resulted in a small and insignificant improvement $(7.4 \pm 6.1 \%, p=0.28$, Cohen's $d=0.33)$. Therefore, the results from both TPE procedures above showed that the enhanced orientation discriminability after training with gratings completely transferred to symmetrical dot patterns, just as the complete transfer in the reverse direction.

One may argue that the observers could covertly practice orientation discrimination with the dot patterns when they performed the easy task during the exposure phase in the TPE experiments. To exclude this possibility, we conducted a control experiment on a new group of observers by replacing the easy dot pattern exposure task (density identification) with a much more demanding one-near-threshold mean luminance discrimina- 
A

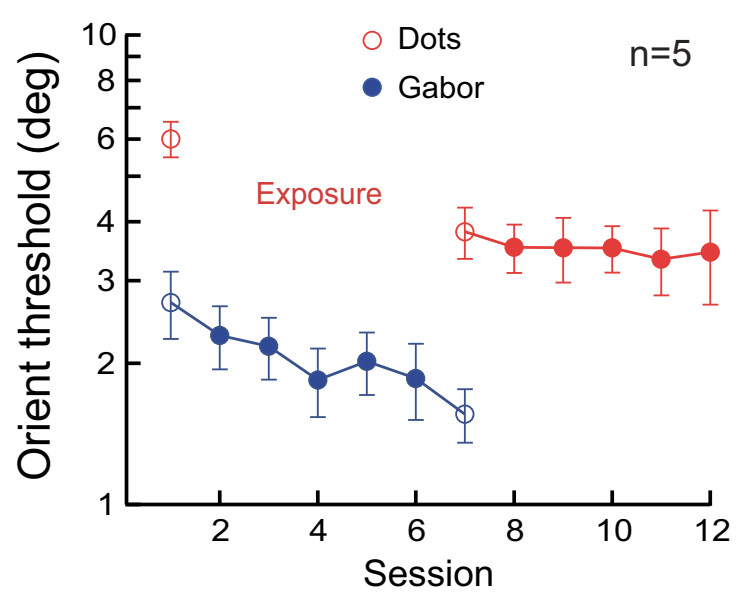

B

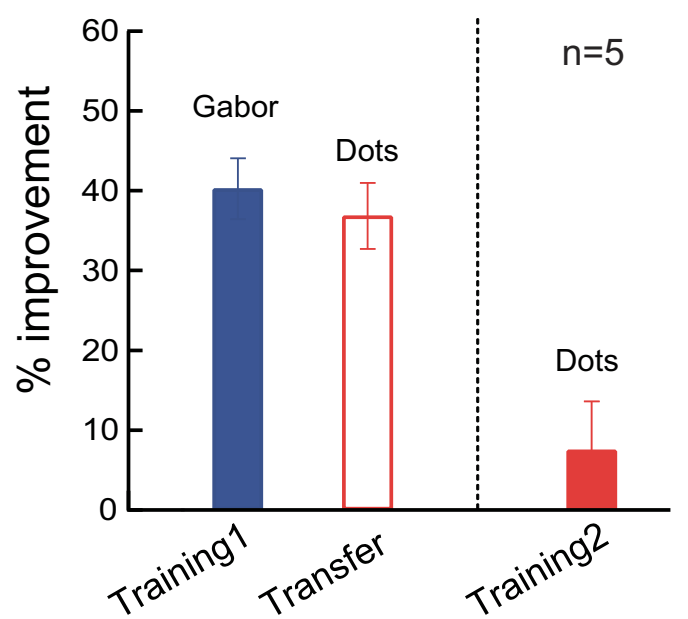

Figure 3. Transfer of orientation discrimination learning from gratings to dots patterns in a simultaneous TPE procedure. $A$, Session-by-session thresholds, first with Gabor orientation training alternated with exposure of dot patterns and then with dot pattern orientation training. B, Summary of grating orientation learning and its transfer to dot patterns ("Training1" and "Transfer"; left two bars) and the impact of further global pattern orientation learning ("Training2"; right bar). Error bars indicate \pm SEM.
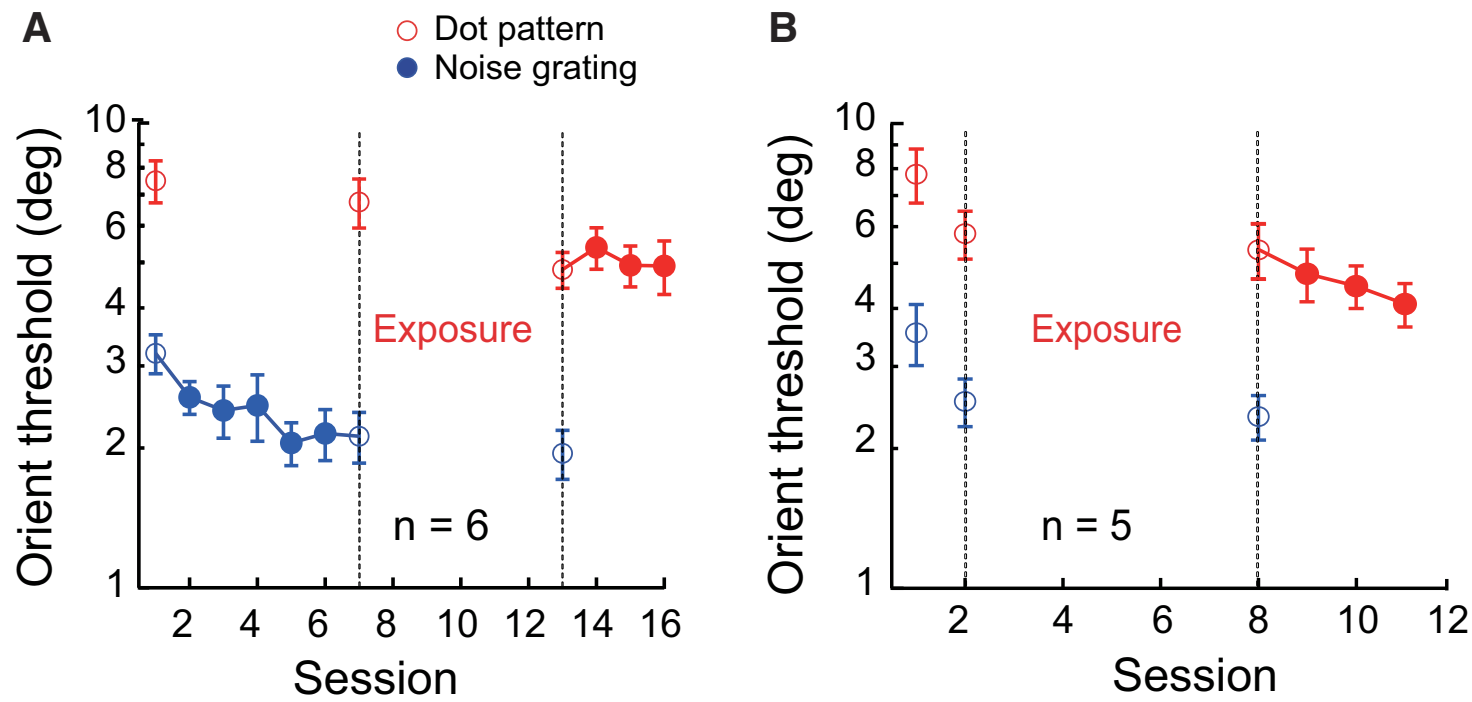

Figure 4. Two control experiments. $A$, TPE enabled learning transfer from grating orientation to dot pattern orientation with a near-threshold exposure task. The experimental design was similar to Figure $2 B$ except that the exposure task (sessions 8-12) was a near-threshold mean luminance discrimination task. $B$, Effect of dot pattern exposure alone. The experimental design was similar to that in $\boldsymbol{A}$ except that there was no initial grating orientation training. Error bars indicate \pm SEM.

tion (see Materials and Methods; Fig. 4A). Initial orientation learning with noise gratings $(34.0 \pm 5.1 \%, p=0.001$, Cohen's $d=2.724)$ had a small and insignificant impact on dot pattern orientation discrimination $(9.7 \pm 7.0 \%, p=0.22$, Cohen's $d=$ $0.57)$, but subsequent dot pattern exposure substantially improved dot pattern orientation discrimination $(26.3 \pm 4.5 \%, p=$ 0.002 , Cohen's $d=2.37$ ). This TPE procedure maximized the observers' discriminability because further direct orientation training with the dot patterns did not further reduce the thresholds $(-0.3 \pm 5.8 \%, p=0.96$, Cohen's $d=0.02)$. These results thus largely excluded a potential contribution of covert orientation learning during the exposure phase.

We showed that TPE enabled learning transfer from gratings to dot patterns, but there was a possibility that the exposure phase alone could improve orientation discrimination with dot patterns. Another control experiment ruled out this possibility. The experimental design was similar to that in Figure $4 A$ except that there was no initial grating orientation training. As shown in Figure $4 B$, mere exposure of the dot patterns (also via a nearthreshold mean luminance discrimination task) had a small and insignificant impact on dot pattern orientation threshold $(8.6 \pm 4.5 \%, p=0.115$, Cohen's $d=0.778)$, but further direct dot pattern orientation training significantly improved dot pattern orientation discrimination $(24.4 \pm 7.3 \%, p=0.029$, Cohen's $d=1.49$ ). Consistent with commonly seen task specificity of perceptual learning, these control data indicate that stimulus exposure alone cannot cause a learning effect comparable to direct training. Therefore, the further improved dot pattern orientation discrimination performance after TPE was more likely due to an interaction between exposure and earlier grating orientation training, which led to improved perceptual sensitivity rather than some general exposure-based learning. 


\section{A}

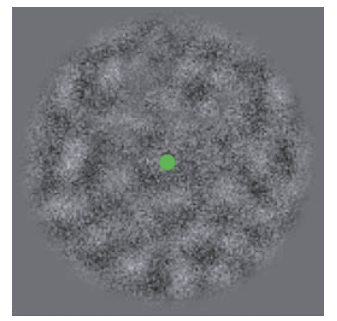

B

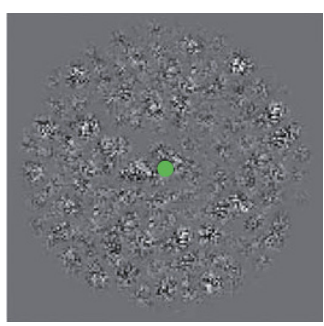

C

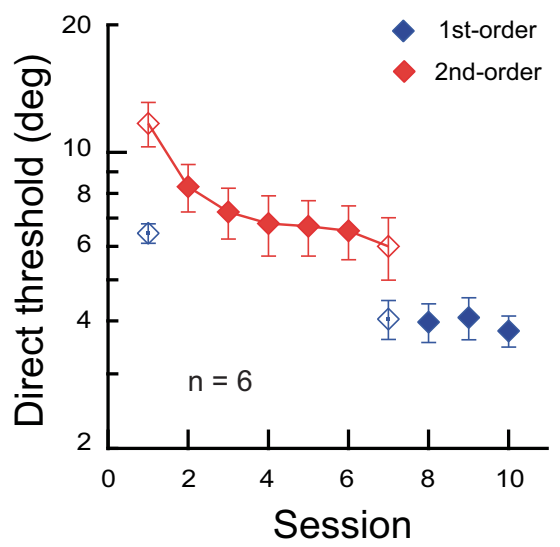

D

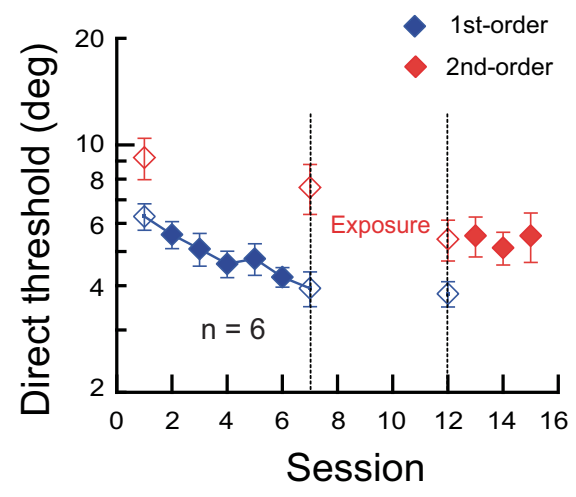

F

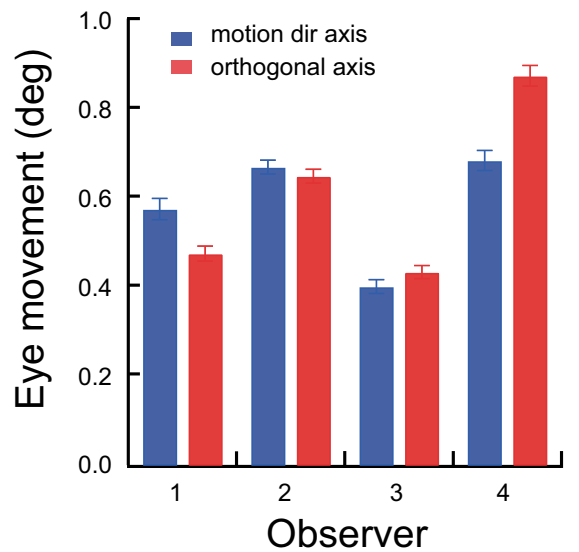

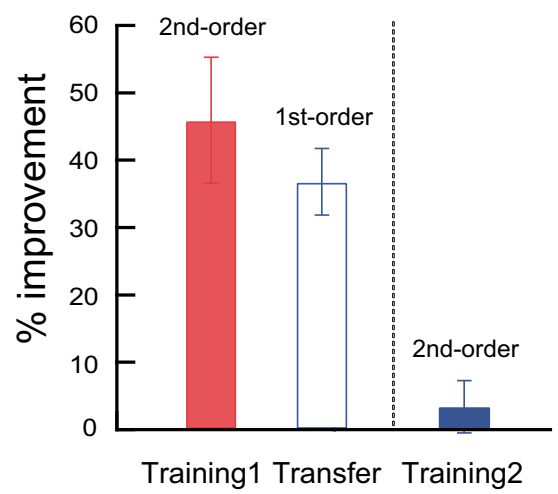

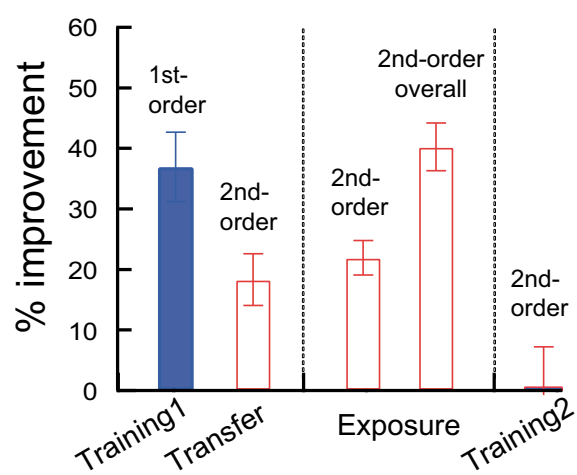

E

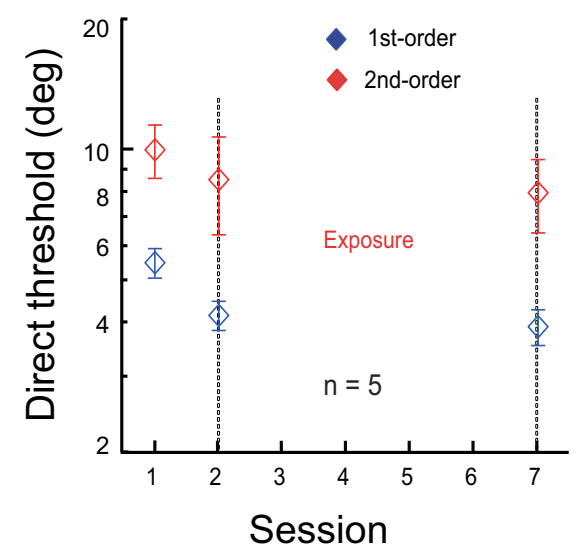

G

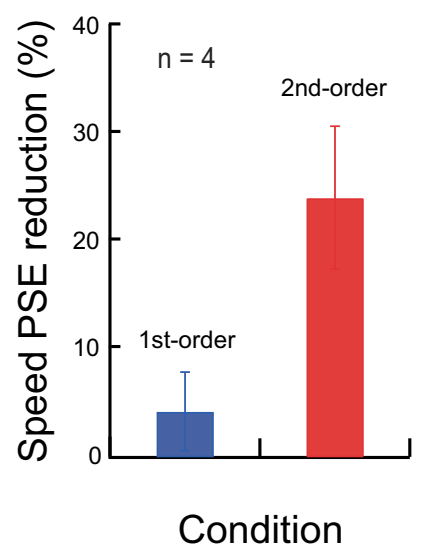

Figure 5. Transfer of motion direction learning between first- and second-order motion signals. $A$, One sample frame of the first-order (luminance-modulated) motion stimulus. The green dot served as a fixation through the trial. B, One sample frame of the second-order (contrast-modulated) motion stimulus. C, Left, Session-by-session thresholds. Right, Learning of second-order motion direction ("Training1") and its transfer to first-order motion stimuli ("Transfer"), as well as the effect of further training with the first-order stimuli ("Training2"). D, Left, Session-by-session thresholds. Right, Learning of first-order motion direction and its transfer to second-order motion stimuli ("Training1" and "Transfer"); the impact of exposure to the second-order stimuli through contrast discrimination training ("Exposure"); and the effect of further training with the second-order stimuli ("Training2"). $\boldsymbol{E}$, Effect of exposure of second-order stimuli alone without initial first-order motion direction training. $\boldsymbol{F}$, Analysis of eye movement patterns in individual observers $(n=4)$ during a training session (10 staircases) of the first-order motion discrimination task. Eye traces during each stimulus presentation were projected to the axes along and orthogonal to the motion direction and the maximal magnitudes of eye movements along these two axes were computed, respectively, and then averaged across trials for each observer. G, Effects of adaptation to the second-order stimulus on the perceived speed of the first- or second-order stimulus. The adaptation effect, or speed PSE reduction, is defined as follows: 100*(baseline_PSE - adaptation_PSE)/baseline_PSE. Error bars indicate \pm SEM.

The same patterns of learning transfer were replicated in motion direction discrimination tasks. The stimuli were luminancemodulated (first-order) motion signals (Fig. 5A) detectable by local linear filters and contrast-modulated (second-order) motion signals (Fig. 5B) detectable only by nonlinear mechanisms
(Lu and Sperling, 2001; Ashida et al., 2007). After 5 sessions of practice (16 blocks of trials per session) with the second-order stimuli, 6 observers improved their direction discrimination of the second-order motion by $45.9 \pm 9.3 \%(p=0.004$, Cohen's $d=2.0$; Fig. $5 C$ ), and the learning significantly transferred to the 
first-order stimuli $(36.8 \pm 4.9 \%, p=0.001$, Cohen's $d=3.0)$. A further 3 sessions of training with the first-order stimuli did not produce additional improvement $(5.3 \pm 3.7 \%, p=0.22$, Cohen's $d=0.58$ ), suggesting that second-order motion discrimination learning transferred to the first-order motion completely.

Similar to the transfer of orientation discrimination learning from simple gratings to complex dot patterns, motion discrimination learning with the first-order stimuli $(36.9 \pm 5.7 \%, p=$ 0.001 , Cohen's $d=2.6$; Fig. $5 D$ ) in 6 observers initially only partially transferred to the second-order stimuli moving at the same direction $(18.3 \pm 4.3 \%, p=0.008$, Cohen's $d=1.76)$. This asymmetric learning transfer between first- and second-order motion was also reported previously (Petrov and Hayes, 2010). Nevertheless, after having the observers exposed to the secondorder motion stimuli in a near-threshold contrast discrimination task (see Materials and Methods) for 5 sessions (16 blocks of 50-60 trials per session), their mean threshold for discriminating the second-order motion direction was further reduced by $22.0 \pm$ $2.9 \%(p<0.001$, Cohen's $d=3.1)$, with a total improvement of $40.3 \pm 4.0 \%$. Three sessions of subsequent direct training with the second-order stimuli did not induce further improvement $(-0.2 \pm 6.0 \%, p=0.75$, Cohen's $d=-0.15)$, indicating that motion discrimination learning had completely transferred from the first- to the second-order stimuli.

A control experiment on another 5 naive observers showed that the exposure procedure by itself (contrast discrimination of the second-order motion stimuli for 5 sessions; Fig. 5E) did not produce any significant improvement in motion discrimination $(-1.1 \pm 2.5 \%, p=0.45$, Cohen's $d=-0.20)$. This task specificity of learning also excluded the possibility that the performance improvement with second-order motion direction after the exposure phase was simply a result of exposure-based general learning; rather, it was the interaction between the exposure and the earlier first-order motion direction training that improved the perceptual sensitivity.

A second control experiment investigated whether learning transfer between the first- and second-order motion stimuli could be due to biased eye movements that could generate streaking signals on the retina along the motion axis. Such signals could be similar for both types of stimuli and picked up by the observers for direction judgment. Four new observers repeated the firstorder motion direction discrimination task for one session at both the training and the opposite directions (five staircases each). We calculated, for each subject, the mean eye positional jitter along and orthogonal to the motion axis. No systematically biased eye movements were observed along the motion axis (Fig. $5 F$ ). These data are consistent with an early analysis by Ball and Sekuler (1982) showing that eye movements do not constitute a confounding factor in motion direction learning.

A third control experiment concerned whether the current first- and second-order motion stimuli share the same neuronal mechanisms. Four experienced observers who were unaware of the purpose of the experiment were first adapted to the secondorder motion stimulus and then tested for the adaptation effects on the perceived speed of the first- or second-order stimulus (Fig. $5 G)$. The results showed that second-order stimulus adaptation significantly reduced the PSE (i.e., perceived speed) of the same second-order stimulus by $24.2 \pm 6.2 \%$ ( $p=0.036$, Cohen's $d=$ 1.82). The adaptation had much less impact on the PSE of the first-order stimulus ( $4.3 \pm 3.7 \%, p=0.034$, Cohen's $d=0.59)$ even if second-order motion direction learning can transfer to first-order motion completely in a conventional training procedure without TPE (Fig. 5C). These results suggest that first- and second-order motion stimuli are processed by separate neuronal mechanisms, consistent with brain imaging data (Ashida et al., 2007).

\section{Discussion}

In the current study, we found mutual transfer of orientation or motion direction discrimination learning between stimuli of different physical properties that have been suggested to engage different mechanisms. In particular, fMRI studies (Sasaki et al., 2005; Tyler et al., 2005) have shown that only higher-tier visual areas, especially the lateral occipital cortex ( $\mathrm{LO}$ ), are differentially activated by symmetrical and random dots, whereas the activations in V1 are indistinguishable. Transcranial magnetic stimulation studies also point to $\mathrm{LO}$ as the cortical locus for symmetry detection (Cattaneo et al., 2011; Bona et al., 2014; Bona et al., 2015). Moreover, fMRI studies using an adaptation protocol have provided compelling evidence that separate mechanisms are responsible for processing first- and second-order orientation (Larsson et al., 2006) and motion (Ashida et al., 2007) because cross-adaptation of cortical activation is absent between firstand second-order stimuli. In Figure 5G, we also show that adaptation to the second-order motion stimulus has different impacts on perceived speed of first- and second-order motion stimuli, indicating that the involved mechanisms are largely separable.

Previous VPL studies have investigated learning transfer between physically distinct orientation signals defined by real and illusory lines (Vogels and Orban, 1987) and between motion signals defined by first- and second-order motion stimuli (Petrov and Hayes, 2010; Vaina and Chubb, 2012). These studies either found one-way learning transfer from second- to first-order stimuli (Vogels and Orban, 1987; Petrov and Hayes, 2010), in agreement with our data collected before the additional exposure phase (Figs. $2 B, 3 A, 5 D$ ), or reported no learning transfer at all (Vaina and Chubb, 2012). Vogels and Orban (1987) interpreted the asymmetric transfer as indications of two pathways for orientation processing, one associated with real contours and the other with both real and illusory contours. Petrov and Hayes (2010) and Vaina and Chubb (2012) also interpreted their data as indications of separate mechanisms for first- and second-order motion processing. However, our TPE results demonstrate that the learning transfer between physically distinct orientation or motion stimuli is actually mutual and complete. Therefore, it is the representations of orientation and motion direction at a conceptual level that are improved by perceptual training. In this sense, perceptual learning is similar to category or object learning.

Our conclusion of VPL operating at a conceptual level represents a new understanding of the nature of VPL. Previous studies have shown learning transfer enabled by double training or TPE procedures (Xiao et al., 2008; J.Y. Zhang et al., 2010), as well as learning transfer with easy tasks (Ahissar and Hochstein, 1997; Liu, 1999). These studies used the same physical stimuli to test learning and transfer, so their implications are more limited than our current findings. Conversely, VPL is found to be task specific (Shiu and Pashler, 1992; Ahissar and Hochstein, 1993) even after TPE training (Cong et al., 2016). The task specificity may set the limit of learning transfer such that, even if VPL can operate at a conceptual level, it would not generate to other feature domains. One possible exception is the report by Matthews et al. (1999) that motion direction learning of a single dot can transfer to line orientation discrimination. However, this is not surprising given the available physiological evidence that such dot movement can activate V1 neurons selectively along their preferred orientation axis (Wörgötter and Eysel, 1991). 
Our control experiments (Figs. 4, 5E) excluded the possibility that exposure to high-order stimuli alone could result in perceptual learning, indicating that exposure interacts with training of low-order stimuli to induce learning transfer. However, it remains mysterious exactly what roles the exposure plays in the TPE procedure. Previously, we showed that TPE can enable complete learning transfer to an untrained orthogonal orientation of the same stimulus (J.Y. Zhang et al., 2010). We now have evidence that the conventional orientation specificity could be a result of insufficient bottom-up stimulation of, or top-down attention to, the untrained stimulus orientation. Therefore, the exposure of the orthogonal orientation in the TPE procedure could amend these bottom-up and top-down issues to enable learning transfer (Xiong et al., 2015). Further studies are necessary to elucidate to what extent this understanding can apply to the current study and whether additional factors also come into play.

Our previous findings of learning transfer to untrained locations, orientations, and directions suggest that VPL is rule-based learning in that the learned reweighting rules can be applied to untrained conditions to allow learning transfer (Xiao et al., 2008; Wang et al., 2012, 2014; Zhang and Yang, 2014; Xiong et al., 2016). Our current data prompt us to speculate that the rules learned and refined from perceptual training must be statistical in nature. For example, they are responsible for assigning a weight to a neuron's response on the basis of its standard score in a distribution of neuronal responses no matter what physical stimulus activates these sensory responses and what cortical level of neurons encode the stimulus. Consistent with this possibility, our data show that, although operating at very different precision levels, training on coarser and higher-order signals leads to a similar amount of improvement in finer discrimination of lowerorder signals ( $42.8 \%$ vs $35.1 \%$ for orientation learning, $p=0.12$, Cohen's $d=0.44 ; 45.9 \%$ vs $36.8 \%$ for motion learning, $p=0.22$, Cohen's $d=0.37$; Figs. $1 B, C, 5 C$ ). The same is true when training in the reverse order using the TPE procedure $(40.1 \%$ vs $35.0 \%$ for orientation learning, $p=0.30$, Cohen's $d=0.29 ; 36.9 \%$ vs $40.3 \%$ for motion learning, $p=0.54$, Cohen's $d=0.27$; Figs. $2 B, C, 5 D$ ). The mutual learning transfer and the similar improvement rates between physically distinct stimuli implicate the involvement of a more central and universal learning mechanism than sensory encoding and response reweighting. Note that learning the statistical rules of reweighting in perceptual learning may be different from rule learning or statistical learning. Perceptual learning, and thus the learning of statistical rules of reweighting, concerns the differentiation of similar stimuli, whereas the latter concerns the discovery of spatial or temporal regularities in changing events or stimuli.

Our conclusion of VPL at a conceptual level is not necessarily conflicting with the existing neurophysiological and brain imaging data. Although learning-induced changes can be seen as early as in V1 (Yan et al., 2014), the manifestation of these changes critically depends on task-specific top-down influences (Li et al., 2008). Therefore, the hard-wired neural circuitry within V1 does not have to be altered physically; instead, changes in V1 functional connectivities gated by top-down influences could account for learning-induced performance improvement (Li et al., 2004; Ramalingam et al., 2013; Moldakarimov et al., 2014). This interpretation is in agreement with the findings that the location and feature specificity can be removed by double training procedures (Xiao et al., 2008; J.Y. Zhang et al., 2010; T. Zhang et al., 2010; Zhang and Yang, 2014; Xiong et al., 2016) as long as the top-down signals can be remapped to affect visual cortical neurons activated by the untrained stimuli (E. Zhang et al., 2013; G.L. Zhang et al., 2013). In this sense, learning-induced improvement in discrimination ability, as well as its specificities, could be viewed as resulting from a close interaction between visual and high-order cortical areas.

In summary, the current study identifies a conceptual component in perceptual learning of discriminating basic visual features. This finding significantly advances our understanding of the mechanisms underlying VPL. Future studies will focus on the brain mechanisms that realize this abstraction process, which could be shared by traditional category and object learning. Research along this line will help us better understand the general rules and mechanisms underlying various forms of learning.

\section{References}

Ahissar M, Hochstein S (1993) Attentional control of early perceptual learning. Proc Natl Acad Sci U S A 90:5718-5722. CrossRef Medline

Ahissar M, Hochstein S (1997) Task difficulty and the specificity of perceptual learning. Nature 387:401-406. CrossRef Medline

Ashida H, Lingnau A, Wall MB, Smith AT (2007) FMRI adaptation reveals separate mechanisms for first-order and second-order motion. J Neurophysiol 97:1319-1325. Medline

Ball K, Sekuler R (1982) A specific and enduring improvement in visual motion discrimination. Science 218:697-698. CrossRef Medline

Bona S, Herbert A, Toneatto C, Silvanto J, Cattaneo Z (2014) The causal role of the lateral occipital complex in visual mirror symmetry detection and grouping: an fMRI-guided TMS study. Cortex 51:46-55. CrossRef Medline

Bona S, Cattaneo Z, Silvanto J (2015) The causal role of the occipital face area (OFA) and lateral occipital (LO) cortex in symmetry perception. J Neurosci 35:731-738. CrossRef Medline

Bruner JS, Goodnow JJ, Austin GA (1956) A study of thinking. New York: Wiley.

Cattaneo Z, Mattavelli G, Papagno C, Herbert A, Silvanto J (2011) The role of the human extrastriate visual cortex in mirror symmetry discrimination: a TMS-adaptation study. Brain Cogn 77:120-127. CrossRef Medline

Cong LJ, Wang RJ, Yu C, Zhang JY (2016) Perceptual learning of basic visual features remains task specific with training-plus-exposure (TPE) training. J Vis. In press.

Crist RE, Kapadia MK, Westheimer G, Gilbert CD (1997) Perceptual learning of spatial localization: specificity for orientation, position, and context. J Neurophysiol 78:2889-2894. Medline

Dosher BA, Lu ZL (1999) Mechanisms of perceptual learning. Vision Res 39:3197-3221. CrossRef Medline

Fahle M (2002) Introduction. In: Perceptual learning (Fahle M, Poggio T, eds), pp. ix-xx. Cambridge, MA: MIT.

Hubel DH, Wiesel TN (1959) Receptive fields of single neurones in the cat's striate cortex. J Physiol 148:574-591. CrossRef Medline

Hubel DH, Wiesel TN (1962) Receptive fields, binocular interaction and functional architecture in the cat's visual cortex. J Physiol 160:106-154. CrossRef Medline

Karni A, Sagi D (1991) Where practice makes perfect in texture discrimination: evidence for primary visual cortex plasticity. Proc Natl Acad Sci U S A 88:4966-4970. CrossRef Medline

Larsson J, Landy MS, Heeger DJ (2006) Orientation-selective adaptation to first- and second-order patterns in human visual cortex. J Neurophysiol 95:862-881. Medline

Law CT, Gold JI (2009) Reinforcement learning can account for associative and perceptual learning on a visual-decision task. Nat Neurosci 12:655-663. CrossRef Medline

Li W, Piech V, Gilbert CD (2004) Perceptual learning and top-down influences in primary visual cortex. Nat Neurosci 7:651-657. CrossRef Medline

Li W, Piëch V, Gilbert CD (2008) Learning to link visual contours. Neuron 57:442-451. CrossRef Medline

Liu Z (1999) Perceptual learning in motion discrimination that generalizes across motion directions. Proc Natl Acad Sci U S A 96:14085-14087. CrossRef Medline

Lu ZL, Sperling G (2001) Three-systems theory of human visual motion perception: review and update. J Opt Soc Am A Opt Image Sci Vis 18: 2331-2370. CrossRef Medline 
Matthews N, Liu Z, Geesaman BJ, Qian N (1999) Perceptual learning on orientation and direction discrimination. Vision Res 39:3692-3701. CrossRef Medline

Moldakarimov S, Bazhenov M, and Sejnowski TJ (2014) Top-down inputs enhance orientation selectivity in neurons of the primary visual cortex during perceptual learning. PLoS Comput Bio 10(8), e1003770. CrossRef

Mollon JD, Danilova MV (1996) Three remarks on perceptual learning. Spat Vis 10:51-58. CrossRef Medline

Pelli DG (1997) The VideoToolbox software for visual psychophysics: transforming numbers into movies. Spat Vis 10:437-442. CrossRef Medline

Petrov AA, Hayes TR (2010) Asymmetric transfer of perceptual learning of luminance- and contrast-modulated motion. J Vis 10:11. Medline

Ramalingam N, McManus JN, Li W, Gilbert CD (2013) Top-down modulation of lateral interactions in visual cortex. J Neurosci 33:1773-1789. CrossRef Medline

Rouder JN, Ratcliff R (2006) Comparing exemplar- and rule-based theories of categorization. Curr Dir Psychol Sci 15:9-13. CrossRef

Sasaki Y, Vanduffel W, Knutsen T, Tyler C, Tootell R (2005) Symmetry activates extrastriate visual cortex in human and nonhuman primates. Proc Natl Acad Sci U S A 102:3159-3163. CrossRef Medline

Schoups AA, Vogels R, Orban GA (1995) Human perceptual learning in identifying the oblique orientation: retinotopy, orientation specificity and monocularity. J Physiol 483:797-810. CrossRef Medline

Shiu LP, Pashler H (1992) Improvement in line orientation discrimination is retinally local but dependent on cognitive set. Percept Psychophys 52: 582-588. CrossRef Medline

Teich AF, Qian N (2003) Learning and adaptation in a recurrent model of V1 orientation selectivity. J Neurophysiol 89:2086-2100. Medline

Tyler CW, Baseler HA, Kontsevich LL, Likova LT, Wade AR,Wandell BA (2005) Predominantly extra-retinotopic cortical response to pattern symmetry. Neuroimage 24:306-314. CrossRef Medline

Vaina LM, Chubb C (2012) Dissociation of first- and second-order motion systems by perceptual learning. Atten Percept Psychophys 74:1009-1019. CrossRef Medline

Vogels R, Orban GA (1987) Illusory contour orientation discrimination. Vision Res 27:453-467. CrossRef Medline

Wang R, Zhang JY, Klein SA, Levi DM, Yu C (2012) Task relevancy and demand modulate double-training enabled transfer of perceptual learning. Vision Res 61:33-38. CrossRef Medline
Wang R, Zhang JY, Klein SA, Levi DM, Yu C (2014) Vernier perceptual learning transfers to completely untrained retinal locations after double training: a "piggybacking" effect. J Vis 14:12. CrossRef Medline

Watson AB, Barlow HB, Robson JG (1983) What does the eye see best? Nature 302:419-422. CrossRef Medline

Wörgötter F, Eysel UT (1991) Axial responses in visual cortical cells: spatiotemporal mechanisms quantified by Fourier components of cortical tuning curves. Exp Brain Res 83:656-664. Medline

Xiao LQ, Zhang JY, Wang R, Klein SA, Levi DM, Yu C (2008) Complete transfer of perceptual learning across retinal locations enabled by double training. Curr Biol 18:1922-1926. CrossRef Medline

Xiong YZ, Zhang JY, Yu C (2015) Under-stimulation at untrained orientation may explain orientation specificity in perceptual learning. J Vis 15:38. CrossRef Medline

Xiong YZ, Xie XY, Yu C (2015) Location and direction specificity in motion direction learning associated with training with a single-level method of constant stimuli. Vision Res 119:9-15. CrossRef Medline

Yan Y, Rasch MJ, Chen M, Xiang X, Huang M, Wu S, Li W (2014) Perceptual training continuously refines neuronal population codes in primary visual cortex. Nat Neurosci 17:1380-1387. CrossRef Medline

Zhang E, Zhang GL, Li W (2013a) Spatiotopic perceptual learning mediated by retinotopic processing and attentional remapping. Eur J Neurosci 38: 3758-3767. CrossRef Medline

Zhang JY, Yang YX (2014) Perceptual learning of motion direction discrimination transfers to an opposite direction with TPE training. Vision Res 99:93-98. CrossRef Medline

Zhang JY, Zhang GL, Xiao LQ, Klein SA, Levi DM, Yu C (2010a) Rule-based learning explains visual perceptual learning and its specificity and transfer. J Neurosci 30:12323-12328. CrossRef Medline

Zhang JY, Cong LJ, Klein SA, Levi DM, Yu C (2014) Perceptual learning improves adult amblyopic vision through rule-based cognitive compensation. Invest Ophthalmol Vis Sci 55:2020-2030. CrossRef Medline

Zhang T, Xiao LQ, Klein SA, Levi DM, Yu C (2010b) Decoupling location specificity from perceptual learning of orientation discrimination. Vision Res 50:368-374. CrossRef Medline

Zhang GL, Cong LJ, Song Y, Yu C (2013b) ERP P1-N1 changes associated with Vernier perceptual learning and its location specificity and transfer. J Vis 13:19. Medline 